\title{
Protracted systemic illness and interstitial nephritis due to minocycline
}

\author{
S.P. Wilkinson ${ }^{1}$, W.K. Stewart ${ }^{1}$, E.M. Spiers ${ }^{2}$ and J. Pears ${ }^{1}$ \\ Departments of ${ }^{1}$ Medicine and ${ }^{2}$ Pathology, Ninewells Hospital and Medical School, Dundee DD1 9SY, UK.
}

\begin{abstract}
Summary: A severe hypersensitivity-like illness with acute renal failure, lymphadenopathy and skin rash is reported following minocycline treatment in a 16 year old male. Following haemodialysis and steroid therapy his illness remitted, only to recur on withdrawal of the steroids. With further steroid treatment he recovered completely. Lymphocyte function tests, performed in an attempt to positively incriminate minocycline, were inconclusive due to a general suppression of the patient's lymphocytes to in vitro stimulation. Hypersensitivity reactions attributed to minocycline include skin rashes, lymphadenopathy and one previous case of acute interstitial nephritis.
\end{abstract}

\section{Introduction}

Tetracycline medication is known to aggravate azotaemic renal failure through an anti-anabolic action but acute interstitial nephritis with this class of antibiotic is unusual. Acute interstitial nephritis has been reported once ${ }^{1}$ and we report a more prolonged hypersensitivity-like illness following minocycline therapy.

\section{Case report}

A 16 year old male student commenced minocycline $50 \mathrm{mg}$ twice daily for sycosis barbae and acne vulgaris. Two weeks later he developed malaise and rigors (Figure 1). A morbilliform rash developed on face, neck and upper trunk and he vomited twice. After 3 days' illness he was admitted with suspected glandular fever. He had previously suffered a glandular feverlike illness and at that time developed a rash after taking penicillin.

On admission he had moderate lymphadenopathy, was pyrexial $\left(39.4^{\circ} \mathrm{C}\right)$ and normotensive $(120 / 80 \mathrm{mmHg})$. Palatal petechiae were noted. Routine urinalysis revealed ketonuria and proteinuria $(300 \mathrm{mg} / \mathrm{l})$. The minocycline was stopped. He had not taken any other medication.

Initially his pyrexia settled although he remained unwell and the rash extended onto his arms and abdomen. The lymphadenopathy increased and he developed bilateral otitis externa. Investigation showed a leucocytosis, increasing from 5.8 to

Correspondence: S.P. Wilkinson, M.R.C.P., Department of Medicine for the Elderly, Woodend Hospital, Aberdeen AB9 2YS, UK.

Accepted: 20 July 1988
$33.6 \times 10^{9} / 1$ over the first week, with activated lymphocytes present. Serum biochemistry and a chest radiograph were normal. One week after admission he again became pyrexial and developed swelling of his face and neck. He was noted to have an eosinophilia $\left(4 \times 10^{9} / 1\right)$ and a new vasculitic-type rash on his fingertips. He was commenced on intravenous erythromycin and hydrocortisone $(400 \mathrm{mg} /$ day) without apparent effect and these drugs were stopped after 48 hours.

Over the next 10 days he remained pyrexial and during the latter part of this period he developed oliguria with serum urea rising to $52 \mathrm{mmol} / \mathrm{l}$ and creatinine $1260 \mu \mathrm{mol} / 1$, despite the reinstitution of intravenous hydrocortisone $400 \mathrm{mg} /$ day. Urine examination showed red blood cells, granular casts, eosinophils, lymphocytes and proteinuria $(1.5 \mathrm{~g} / 24 \mathrm{~h})$. Renal ultrasonography revealed swollen, smooth kidneys without signs of obstruction. He required haemodialysis via a subclavian catheter on 5 successive days. His urine output gradually improved, renal function recovered and the steroid medication was gradually reduced. When his serum creatinine was $180 \mu \mathrm{mol} / 1$, he was discharged taking prednisolone $5 \mathrm{mg} /$ day, 4 weeks after the onset of his illness.

He was readmitted the following day with a recrudescence of rash and fever. Serum creatinine had risen to $250 \mu \mathrm{mol} / 1$ and ESR from 55 to $140 \mathrm{~mm} / \mathrm{h}$. He was recommenced on oral prednisolone $(60 \mathrm{mg} /$ day $)$ and once again his illness settled. A percutaneous renal biopsy was then performed. This showed features of an interstitial nephritis with an infiltrate of lymphocytes, plasma cells, some neutrophil polymorphs, and very scanty eosinophils in the cortex. The infiltrate extended between the tubular epithelial cells which

(C) The Fellowship of Postgraduate Medicine, 1989 

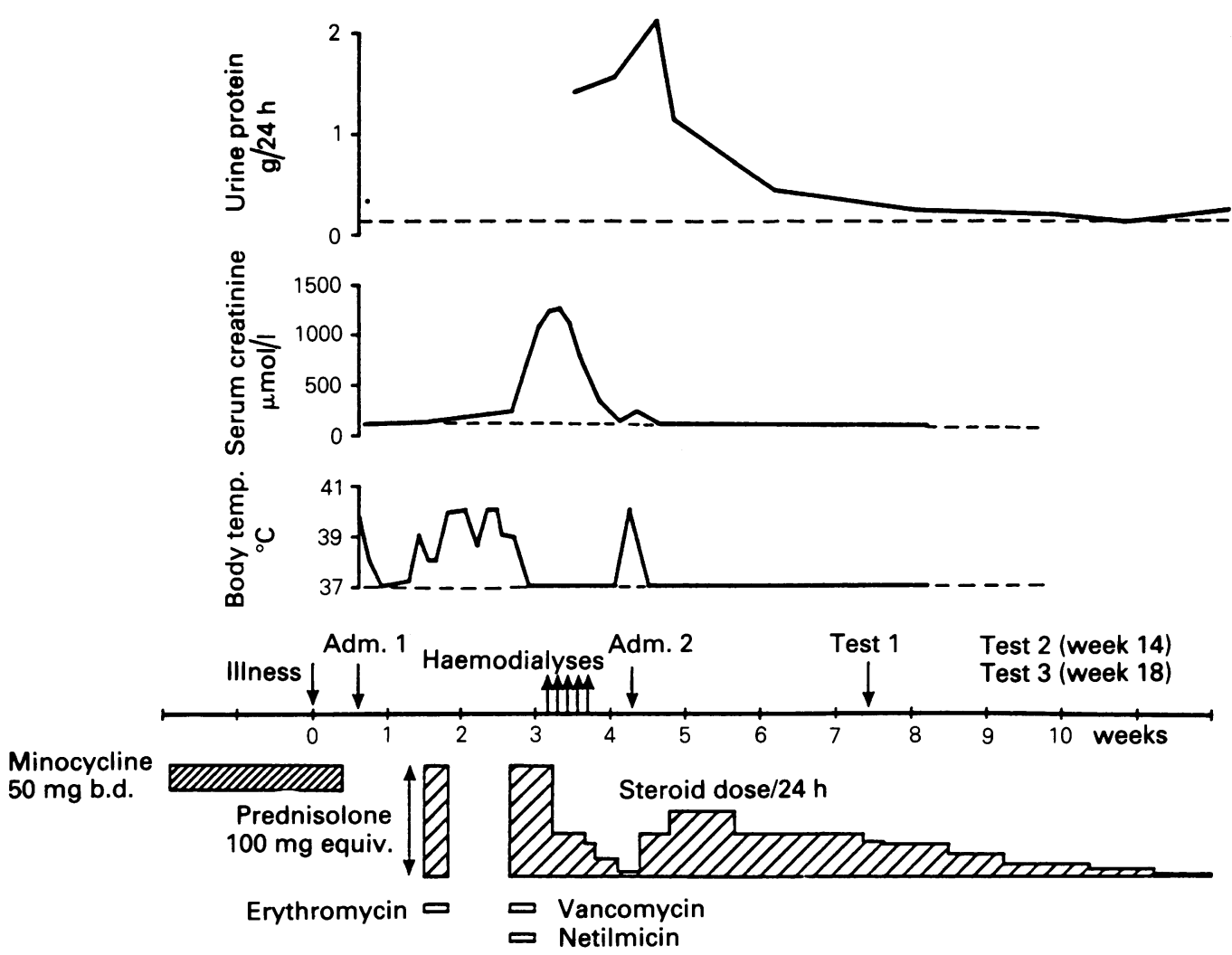

Figure 1 Course of the clinical illness in relation to minocycline and steroid therapy.

showed signs of degeneration and regeneration. The glomeruli showed only slight capsular adhesions. Immunoperoxidase examination was negative. He was discharged from hospital with serum creatinine $90 \mu \mathrm{mol} / \mathrm{l}$ and proteinuria $700 \mathrm{mg} / 24 \mathrm{~h}$. His lymph node enlargement had cleared as had the skin inflammation although generalized skin desquamation continued.

His steroid medication was gradually withdrawn over the subsequent two months. Proteinuria fell to within normal limits $(<150 \mathrm{mg} / 24 \mathrm{~h})$, eosinophiluria disappeared and his renal function returned to normal. He remained well over the next 6 months, but then developed thyrotoxicosis with positive cytoplasmic antibodies. He was treated initially with carbimazole followed by subtotal thyroidectomy.

\section{Investigations}

Negative serological tests during the course of the patient's illness included those for influenza A and B, adenoviruses, varicella-zoster, herpes simplex, par- vovirus, hepatitis $\mathbf{B}$, human immunodeficiency virus, KHF (Hantaan) virus, mycoplasma, Q fever, psittacosis, leptospira and toxoplasma. TPHA, antistreptolysin $O$ titres, rheumatoid and antinuclear factors were also negative. Titres indicated past infection with cytomegalovirus, Epstein-Barr virus and rubella. Blood and throat swab cultures for pathogenic bacteria were negative and throat swabs had no cytopathic effect in cell culture. Stool examination for cysts, ova or protozoa was negative. At the onset of the renal failure immune complex levels were slightly raised and $\mathrm{C}_{4}$ was slightly low on one occasion. $\mathrm{C}_{3}$ was normal throughout.

Attempts were made to positively incriminate minocycline in the delayed-type hypersensitivity reaction, by examining its effect on lymphocytes. The in vitro response of the patient's lymphocytes to mitogen stimulation with phytohaemagglutinin (PHA) and antigenic stimulation with purified protein derivative (PPD) was measured. The tests were performed both in the absence and in the presence of concentrations of minocycline equivalent to blood levels within the therapeutic range. The response was determined by 
measurement of tritiated thymidine uptake at 72 hours (with PHA stimulation) and at 5 days (with PPD stimulation); thymidine uptake is a measure of replicative growth. ${ }^{2}$

These tests were repeated on three occasions (during weeks 7,14 and 18 of the illness). There was no significant difference between the patient and control subject in lymphocyte response (expressed as a percentage of response without minocycline) to increasing concentrations of minocycline. The response of the patient's lymphocytes to PHA was suppressed on first testing but normal on the second occasion. Response to PPD was suppressed on repeated testing. The response of lymphocytes from the normal subject to PHA and PPD was suppressed by plasma from the patient (PHA during, and PPD during and after the period of steroid medication) whilst the patient's cells were more responsive when suspended in normal plasma.

Lymphocyte marker studies revealed an excess of $T$ cells, the increase being due to $T$ helper cells. B cell and $\mathrm{T}$ suppressor cell numbers were normal.

\section{Discussion}

Hypersensitivity-mediated interstitial nephritis is a recognized but rare concomitant of systemic antibiotic therapy, the most common association being with methicillin and ampicillin. ${ }^{3}$ Our patient's presentation was initially typical of an antibiotic-associated hypersensitivity reaction with rash and fever developing within 14 days of exposure to the drug. ${ }^{3}$ However, although mild proteinuria was present on admission, oliguric renal failure did not develop until 17 days later. During this interval his disease was active as evidenced by worsening lymphadenopathy and skin rash. At the onset of renal failure, features typical of interstitial nephritis included smooth, swollen kidneys on ultrasound and eosinophiluria. ${ }^{3,4}$ Many infectious agents may lead to the development of allergic interstitial nephritis. ${ }^{5}$ We were, however, unable to detect any evidence of recent infection in our patient.

Although acute interstitial nephritis associated with minocycline medication has been reported only once before, ${ }^{1}$ the Committee on Safety of Medicines has received six reports of skin rash and one of lymphadenopathy. Hyperpigmentation appears to be the most common cutaneous reaction, ${ }^{5}$ although fixed drug eruptions and Stevens-Johnson syndrome ${ }^{7}$ have been reported.
Several immunological mechanisms have been proposed to account for acute interstitial nephritis. These include cell-mediated immunity, IgG autoantibodies and immune complex deposition, and involvement of reaginic antibodies. ${ }^{3,8}$ In the one previous case of minocycline-induced interstitial nephritis the course of the illness was both less severe and shorter-lived, resolving without treatment. In that patient, immunofluorescence studies of the renal biopsy specimen were positive for $\operatorname{IgA}$ and $\mathrm{C}_{3}{ }^{1}$ There was no evidence of immunoreactants on immunoperoxidase examination of our patient's renal biopsy. The finding of slightly raised immune complex levels at the height of his illness does, however, suggest some involvement of a humoral mechanism. It is interesting to speculate that the minocycline may have allowed the subsequent development of thyrotoxicosis in this young man, by some modulation of immune mechanisms.

Lymphocyte stimulation tests have been used occasionally in patients with hypersensitivity interstitial nephritis in an attempt to incriminate the putative drug positively, without the dangers of rechallenge. ${ }^{9,10}$ We were unfortunately unable to demonstrate in vitro lymphocyte stimulation by minocycline in our patient. There are two possible explanations for this. Firstly, minocycline has been shown to suppress mitogen-stimulated lymphocyte growth in the higher concentrations used in our test. ${ }^{2}$ This was confirmed by our data. Secondly, our patient showed general suppression of lymphocyte response to stimulants compared to a normal subject (and compared to the laboratory normal range in the case of PPD). This reduced responsiveness seemed to be mediated, at least in part, by a factor in his plasma.

The time course of our patient's illness (at least 6 weeks), as well as some of the clinical features, are similar to those of a case of diphenylhydantoininduced interstitial nephritis reported by Matson et $a l .^{10}$ Their patient also displayed generally suppressed lymphocyte responsiveness but had a positive stimulation test with diphenylhydantoin.

The role of steroid therapy in acute drug-induced interstitial nephritis is unproved ${ }^{11}$ although there are many anecdotal reports of its value. ${ }^{3,4}$ In our patient the evidence would suggest that it was beneficial.

\section{Acknowledgement}

We thank Dr C. Pennington for permission to report this case. 


\section{References}

1. Walker, R.G., Thomson, N.M., Dowling, J.P. \& Ogg, C.S. Minocycline-induced acute interstitial nephritis. $\mathrm{Br}$ Med J 1979, 1: 524.

2. Potts, R.C., MacConnachie, A., Brown, R.A. et al. Some tetracycline drugs suppress mitogen-stimulated lymphocyte growth but others do not. Br J Clin Pharmacol 1983, 16: $127-132$.

3. Pusey, C.D., Saltissi, D., Bloodworth, L., Rainford, D.J. \& Christie, J.L. Drug associated acute interstitial nephritis: clinical and pathological features and the response to high dose steroid therapy. $Q J$ Med 1983, 52: 194-211.

4. Galpin, J.E., Shinaberger, J.H., Stanley, T.M. et al. Acute interstitial nephritis due to methicillin. Am J Med 1978, 65: 756-765.

5. Cameron, J.S. Allergic interstitial nephritis: clinical features and pathogenesis. $Q J$ Med 1988, 66: 97-115.

6. Argenyi, Z.B., Finelli, L., Bergfeld, W.F. et al. Minocycline-related cutaneous hyperpigmentation as demonstrated by light microscopy, electron microscopy and X-ray energy spectroscopy. J Cut Pathol 1987, 14: $176-180$.
7. Shoji, A., Sormeda, Y. \& Hamada, T. Stevens-Johnson syndrome due to minocycline therapy (letter). Arch Dermatol 1987, 123: 18-20.

8. McLuskey, R.T. \& Colvin, R.B. Immunological aspects of renal tubular and interstitial diseases. Ann Rev Med 1978, 29: 191-203.

9. Sheth, K.J., Casper, J.T. \& Good, T.A. Interstitial nephritis due to phenytoin hypersensitivity. $J$ Pediatr 1977, 91: 438-441.

10. Matson, J.R., Krous, H.F. \& Blackstock, R. Diphenylhydantoin-induced hypersensitivity reaction with interstitial nephritis. Hum Pathol 1985, 16: 94-97.

11. Appel, G.B. A decade of penicillin related interstitial nephritis - more questions than answers. Clin Nephrol 1980, 13: 151-154. 